\title{
Erythroplasia: the oral epithelial lesion with the greatest potential for malignant transformation - a mini review
}

\begin{abstract}
The purpose of this mini review was to provide information to improve the knowledge of dental surgeons and researchers about oral erythroplasia. It is defined as a red spot or plaque that cannot be diagnosed clinically or histopathologically as any other condition. Its pathogenesis process is unknown. Clinically, the lesions may present in different shapes and sizes and the preference sites for its affection are floor of the mouth, tongue, soft palate. Erytrhroplasia is rare, but has been considered, among of the potentially premalignant oral epithelial lesions, the one with the greatest potential for malignant transformation. Despite the severity, few studies evaluating erythroplasia alone. New methods of diagnosis and treatment have been studied, however, clinical and histopathological examination is still diagnostic methods, and the degree of epithelial dysplasia guides the treatment. However, less subjective alternatives for determining the degree of severity of oral dysplasias need to be established.
\end{abstract}

Keywords: erythroplasia; malignant; oral squamous cell carcinoma

Volume 9 Issue 6 - 2018

\author{
Arieli Carini Michels,' Suelen Teixeira \\ Luiz,' Emanuela Carla dos Santos,' Antônio \\ Adilson Soares de Lima, ${ }^{2}$ Patrícia Carlos \\ Caldeira, ${ }^{3}$ Aldini Beuting Pereira Kitahara,' \\ Ana Maria Trindade Grégio Hardy,' Luciana \\ Reis Azevedo Alanis,' Aline Cristina Batista \\ Rodrigues Johann'
}

'Pontifical Catholic University of Paraná, Brazil

${ }^{2}$ Federal University of Paraná, Brazil

Correspondence: Arieli Michels, Pontifical Catholic University of Paraná, Brazil, Tel +5547988903897,

Email ariali_eli@yahoo.com.br

Received: November 0I, 2018 | Published: December 13, 2018

\section{Introduction}

The term oral potentially malignant disorders renamed potentially premalignant oral epithelial lesions, ${ }^{1}$ are presentations that implicate a risk of cancer development, whether in a precursor lesion or in clinically normal oral mucosa. Therefore, despite the unknown number of cases, ${ }^{2}$ oral cancer may arise from these lesions. ${ }^{3}$ Most of these lesions may be asymptomatic at the beginning in their evolution and may be detected by dentists on routine oral examination, being necessary these professionals to know about the clinical features and diagnostic aspects of these lesions. ${ }^{4}$ Among potentially premalignant oral epithelial lesions there is erythroplasia that has been described as a red spot or plaque that cannot be diagnosed clinically or histopathologically as any other condition..$^{5-7}$ Historically, it is known that Queyrat, in 1911, have been describe erythroplasia lesions affecting the region of the glans penis, and representing malignant potential, but it is unknown the period these lesions, which resemble clinically and histopathologically, began to be described affecting the region of the oral mucosa. ${ }^{8}$

\section{Etiology}

Smoking and alcohol consumption are associated with the presence of oral lesions, ${ }^{6,7,910}$ presenting as important etiological factors, In addition, one study reported the association of oral erythroplasia with Human Papillomavirus infection, observed in 25\% erythroplasia cases in gutka chewers of Karachi ${ }^{11}$, but it is still unknown whether Human Papillomavirus infection is an etiologic factor or a coincident event of erythroplasia. ${ }^{12}$ The lesions pathogenesis process is yet unknown. ${ }^{6,7,9,10}$

\section{Clinical characteristics}

Clinically, erythroplasia lesions appear as bright red, velvety patches or plaques, that may be flat or depressed, ${ }^{5,8}$ and are, generally, asymptomatic, but in some cases associated with burning sensation. ${ }^{13}$ The lesions of erythroplasia are usually irregular in outline, although well defined, and have a bright red velvety surface. Occasionally, the surface is granular. ${ }^{4}$ The preference sites for its affection are floor of the mouth, tongue, soft palate. ${ }^{9,13}$ May be present in any region of the oral mucosa, usually as a single lesion ${ }^{5}$. When it comes to extension, lesions smaller than 1.5 centimeters in diameter are more common, however, they may reach larger proportions, exceeding 4 centimeters. ${ }^{5}$

\section{Epidemiological characteristics}

Oral erythroplasia is rare, $,, 8,10,13,14$ and a prevalence of 0.02 to $0.83 \%$ has been estimated ${ }^{5,8}$. Despite its rarity, among of the potentially premalignant oral epithelial lesions, the erythroplasia is considered the one with the greatest potential for malignant transformation. ${ }^{8,10,14}$ The lesions are most frequently identified in males between the 6 th and 8th decades of life..$^{5,10,13}$

\section{Histopathological characteristics}

Histopathologically, erythroplasias' epithelium is thin and atrophic, allowing visualization of the underlying microcirculation (producing a red appearance). ${ }^{8,15}$ Erythroplasia may present with different degrees of intraepithelial dysplasia that consisting architectural and cytologic disturbances and are a consistent finding in these lesions. ${ }^{2}$ Classically, dysplasia is divided into grades of severity, according of thirds of oral epithelium affected. In erythroplasia are most commonly seen: moderate (dysplasia by extension into middle third) and severe (dysplasia extension into upper third), ${ }^{5,6}$ or carcinoma in situ (full or almost full thickness architectural abnormalities in the viable cellular layers accompanied by pronounced cytologic atypia, but invasion is not present ${ }^{16}$ or invasive carcinoma. ${ }^{8}$ It is important to note that the dysplasia classification of erythroplasia evidenced in the previous studies is according to the old World Health Organization classification (2005). ${ }^{16}$ Currently, carcinoma in situ is synonymous with severe dysplasia. ${ }^{2}$ Besides that, the classification of dysplasia is poorly reproducible among observers, consequently other forms of classification, such as the binary system, ${ }^{17,18}$ have been suggested. However, according to World Health Organization, validation of this system is necessary for the oral cavity before it can be routinely applied to this region. ${ }^{2}$ 


\section{Diagnosis}

Histopathological patterns, varying dysplasia's degrees or carcinomas, were not correlated with the clinical presentation of oral lesions, therefore, biopsy, followed by histopathological analysis, is the only method to determine epithelial tissue's conditions. ${ }^{4,8,14}$ In a recent study using Narrow-Band Imaging, it was possible to establish that elongated and destructive intraepithelial microvascularization patterns are important indicators for the detection of high-grade dysplasia, carcinoma in situ, and invasive carcinoma in oral erythroplasia, which could be suggestive for non-invasive diagnostic methods. However, the authors indicate the need for future largescale and multicenter studies to definitely conclusion. ${ }^{8}$ In addition, the autofluorescence, vital staining (toluidine blue, Lugol's iodine), and brush cytology are also minimally invasive techniques and have practically no morbidity but present a considerable false-positive and false-negative rate, being, therefore, only aids to physical examination and biopsy, which are still considered as the gold standard for the detection and diagnosis. ${ }^{1}$

\section{Differential diagnosis}

Oral erythroplasia is an exclusion diagnosis, in this way, it is necessary to differentiate it from other oral conditions that present with red coloration. Among the reddish oral conditions are local irritations, or infections such as candidiasis, histoplasmosis, tuberculosis. In addition, it should be differentiated from erosive lichen planus, lupus erythematosus, pemphigus, pemphigoid, telangiectasia, Kaposi's sarcoma, mucositis and others that may present similar characteristics. Well-demarcated, solitary presentation of erythroplasia helps clinically distinguish it from the other more widespread lesions listed above. ${ }^{4}$ However, because of the appearance and, mainly, the similar clinical behavior among some lesions, biopsy and histopathological examination are necessary to confirm the diagnosis, that should be undertaken urgently because the erythroplasia may be a severe dysplasia or may harbor carcinoma in situ or invasive carcinoma. ${ }^{4}$

\section{Malignant transformation}

Most erythroplasias have evidence of invasive carcinoma or carcinoma in situ, so progression to malignancy is debatable, because it will probably be treated as malignant at the first presentation. If it is not cancer at the first biopsy, it will present histological characteristics of high risk, thus, the best estimates for the malignant progression can be derived from data for the transformation of lesions that present severe epithelial dysplasia. ${ }^{19}$

\section{Treatment}

The management of premalignant lesions is complex, and the literature regarding the ideal treatment is conflicting. The progression from normal mucosa to premalignant or dysplastic mucosa and to finally malignant change is a complex interplay between the environment and the host. ${ }^{1}$ The histopathological examination performed after the biopsy will determine the erythroplasia treatment. As previously seen, the diagnosis of moderate or severe dysplasia and carcinoma in situ are frequent, in these cases, the treatment of choice is excision either by cold knife cone or by laser. ${ }^{6,10,15}$ In addition, because of the high risk of malignant transformation, it is important to periodically follow up the patients, through consultation and examination of the mucous membranes, as well as the elimination of smoking habits and alcoholism..$^{6,10}$ The choice of treatment for invasive carcinoma is based on the clinical staging. ${ }^{13}$ Malignancy transformation rate for oral carcinoma in situ and/or severe epithelial dysplasia varies 14.3 to $50 \% .^{20} \mathrm{~A}$ recent systematic review of topical treatments of oral potentially malignant disorders, including erythroplasia, has revealed that there are few studies identifying these therapeutic approaches. The authors indicate the need for randomized placebo controlled trials and long-term follow-up to be able to perform better potentially malignant disorders treatments, as well as to prevent malignant transformation. ${ }^{21}$

\section{Conclusion}

Despite the severity, few studies evaluating erythroplasia alone. New methods of diagnosis and treatment, both less invasive, have been studied, however, clinical and histopathological examination is still diagnostic methods, and the degree of epithelial dysplasia guides the treatment. Still, less subjective alternatives for determining the degree of severity of oral dysplasia need to be established.

\section{Acknowledgments}

None.

\section{Conflicts of interest}

The authors declare that there is no conflict of interest.

\section{References}

1. Awadallah M, Idle M, Patel K, et al. Management update of potentially premalignant oral epithelial lesions. Oral Surg Oral Med Oral Pathol Oral Radiol. 2018;125(6):628-636.

2. Reibel J, Gale N, Hille J et al. Oral Potentially malignant disorders and oral epithelial dysplasia. In: El-Nagar AK, Chan JKC, Grandis JR, Takata T, Slootweg PJ, editors. WHO classification Head and Neck Tumours. Lyon: IARC Press;2017:112-114.

3. Kumar YS, Acharya S, Pentapati KC. Prevalence of oral potentially malignant disorders in workers of Udupi taluk. South Asian J Cancer. 2015;4(3):130-133.

4. Warnakulasuriya S. Clinical features and presentation of oral potentially malignant disorders. Oral Surg Oral Med Oral Pathol Oral Radiol. 2018;125(6):582-590.

5. Yardimci G, Kutlubay Z, Engin B, et al. Precancerous lesions of oral mucosa. World J Clin Cases. 2014;2(12):866-872.

6. van der Waal I. Potentially malignant disorders of the oral and oropharyngeal mucosa; present concepts of management. Oral Oncol. 2010;46(6):423-435.

7. Warnakulasuriya S, Johnson NW, Van DW I. Nomenclature and classification of potentially malignant disorders of the oral mucosa. $J$ Oral Pathol Med. 2007;36(10):575-580.

8. Yang SW, Lee YS, Chang LC, et al. Clinical characteristics of narrowband imaging of oral Erythroplasia and its correlation with pathology. BMC Cancer. 2015;15:406.

9. Villa A, Villa C, Abati S. Oral cancer and oral Erythroplasia: an update and implication for clinicians. Aust Dent J. 2011;56(3):253-256.

10. Hosni ES, Salum FG, Cherubini K, et al. Oral Erythroplasia and speckled leukoplakia: retrospective analysis of 13 cases. Braz J Otorhinolaryngol. 2009;75(2):295-299.

11. Baig S, Lucky MH, Qamar A, et al. Human papilloma virus and oral lesions in gutka eating subjects in Karachi. J Coll Physicians Surg Pak. 2012;22(3):135-138.

12. Chen $\mathrm{X}$, Zhao Y. Human papillomavirus infection in oral potentially malignant disorders and cancer. Arch Oral Biol. 2017;83:334-339. 
13. Mortazavi H, Baharvand M, Mehdipour M. Oral potentially malignant disorders: an overview of more than 20 entities. J Dent Res Dent Clin Dent Prospects. 2014;8(1):6-14.

14. van der Waal I. Oral potentially malignant disorders: is malignant transformation predictable and preventable? Med Oral Patol Oral Cir Bucal. 2014;19(4):e386-90.

15. Yang SW, Lee YS, Chang LC, et al. Outcome of excision of oral Erythroplasia. Br J Oral Maxillofac Surg. 2015;53(2):142-147.

16. Gale N, Pilch BZ, Sidransky D, et al. Epithelial precursor lesions. In Barnes L, Eveson JW, Reichart P, Sidransky D, editors. World health organization classification of tumours. Pathology \& Genetics Head and Neck Tumours. Lyon: IARC Press;2005:177-179.

17. Kujan O, Oliver RJ, Khattab A, et al. Evaluation of a new binary system of grading oral epithelial dysplasia for prediction of malignant transformation. Oral Oncol. 2006;42(10):987-993.
18. Nankivell P, Williams H, Matthews $\mathrm{P}$, et al. O sistema binário de classificação da displasia oral: teste de validade e sugeriu melhoria. Oral Surg Oral Med Oral Pathol Oral Radiol. 2013;115(1):87-94.

19. Speight PM, Khurram SA, Kujan O. Oral potentially malignant disorders: risk ofprogression to malignancy. Oral Surg Oral Med Oral Pathol Oral Radiol. 2018;125(6):612-627.

20. Reichart PA, Philipsen HP. Oral Erythroplasia--a review. Oral Oncol. 2005;41(6):551-161.

21. Chau L, Jabara JT, Lai W, et al. Topical agents for oral cancer chemoprevention: A systematic review of the literature. Oral Oncol. 2017;67:153-159. 